\title{
Real-time bioluminescence and tomographic imaging of gastric cancer in a novel orthotopic mouse model
}

\author{
HAO HU ${ }^{1 *}$, JUNTING LIU $^{2 *}$, LIPING YAO ${ }^{1 *}$, JIPENG YIN $^{1}$, NING SU$^{4}$, XIANGQIANG LIU ${ }^{1}$, \\ FENG CAO $^{3}$, JIMIN LIANG ${ }^{2}$, YONGZHAN NIE ${ }^{1}$ and KAICHUN WU ${ }^{1}$
}

\begin{abstract}
${ }^{1}$ State Key Laboratory of Cancer Biology, Xijing Hospital of Digestive Diseases, Fourth Military Medical University, Xi'an 710032; ${ }^{2}$ Life Sciences Research Center, School of Life Sciences and Technology, Xidian University, Xi'an 710071; Departments of ${ }^{3}$ Cardiology, ${ }^{4}$ Neurosurgery, Xijing Hospital, Fourth Military Medical University, Xi'an 710032, P.R. China
\end{abstract}

Received September 23, 2011; Accepted November 14, 2011

DOI: $10.3892 / o r .2012 .1713$

\begin{abstract}
Gastric cancer is the second leading cause of cancer mortality worldwide. Understanding the multistep process of carcinogenesis of gastric cancer is pivotal to develop novel therapeutic strategies. Molecular imaging in preclinical cancer models bridges the gap of laboratory-based experiment and clinical translation. To this end, the human gastric cancer cell line SGC-7901 was established to stably express luciferase and GFP by lentiviral transduction (SGC7901Luc-GFP). Preclinical models were developed by orthotopic transplantation of SGC-7901-Luc-GFP into the sub-serosal layer of the stomach of immunocompromised mice. Tumor progression and therapeutic responses were dynamically tracked by bioluminescence imaging (BLI). Bioluminescence tomography (BLT) was used to monitor stereoscopic morphological and signal changes during tumor progression. Good correlation between cell number and bioluminescence/ fluorescence intensity was observed $\left(\mathrm{R}^{2}=0.9983 / \mathrm{r}^{2}=0.9974\right)$ in vitro. Tumor progression and therapeutic response could be successfully followed directly by BLI. Importantly, BLT provided a more accurate spatial location and tomographic quantification of the internal lesion. In conclusion, our novel bioluminescence-based preclinical gastric cancer models enable superior, noninvasive monitoring gastric cancer progression and their drug responses. The BLT technique in particular, may have great potential for future oncological studies.
\end{abstract}

Correspondence to: Dr Kaichun Wu, State Key Laboratory of Cancer Biology, Xijing Hospital of Digestive Diseases, Fourth Military Medical University, Xi'an 710032, P.R. China

E-mail: kaicwu@fmmu.edu.cn

${ }^{*}$ Contributed equally

Key words: preclinical cancer model, orthotopic implantation, bioluminescence tomography, bioluminescence imaging, threedimensional reconstruction, drug efficacy evaluation

\section{Introduction}

Gastric cancer is the second leading cause of cancer mortality worldwide with a 5-year survival rate of $20-30 \%$ for the advanced disease $(1,2)$. Therefore, understanding the multistep of carcinogenesis of gastric cancer is a pivotal to develop novel therapeutic strategy.

Currently, molecular imaging technology which allows for non-invasive and longitudinal observation has become an invaluable tool for cancer preclinical studies $(3,4)$. Bioluminescence imaging (BLI), especially, demonstrates the most desirable combination of high sensitivity and easy handling (5) and is suitable for acquire signal deep in tissue (6). It has been widely used for monitoring different cancer biological processes [angiogenesis $(7,8)$, infinite proliferation and metastasis $(9,10)$, multidrug resistance $(11,12)]$ and assessing the efficacy of different therapeutic modalities (13-15).

Rodent cancer model is the essential link between laboratory-based experiments and clinical translation of novel therapeutic strategies. Compared with ectopic cancer models, such as subcutaneous, experimental or metastasis models, orthotopic implantation has some unique advantages. Firstly, it preserves the maximum extent of the cancer 'nature' microenvironment (16-18). In addition, the orthotopic cancer model reflects a relevant site to assess therapeutic outcomes, avoiding the false-positive drug responses caused by other cancer models (19). Although the orthotopic gastric cancer model has been employed in previous studies (20-22), little attention has been paid to its direct use for in vivo longitudinal monitoring and drug evaluation.

In recent years, the development of new biomedical imaging technology has witnessed discontent with the BLI technique, which only provides 2-dimensional information. Therefore, a more accurate anatomical location is required to describe the cancerous lesion in a whole-body view. Combined with BLT and micro-CT, hybrid bioluminescence tomography (BLT) is able to provide both source localization and distribution inside a living animal, which produces accurate tomographic reconstructions and visualization of the cancer lesion in a 3-dimentional (3D) mode $(23,24)$. To the best of our knowledge, few studies have been done on 3D reconstruction of an orthotopic gastric tumor with molecular imaging. 
In this study, human gastric cancer cell line SGC7901 was established to stably express luciferase and GFP by lentiviral transduction (SGC7901-Luc-GFP). This cell subsequently was used to develop novel preclinical models of gastric cancer. Our model enabled sensitive detection of tumor progression and therapeutic responses by bioluminescence imaging (BLI). In addition, bioluminescent tomography (BLT) can successfully monitor stereoscopic morphological and signal changes during tumor progression.

\section{Materials and methods}

Cell culture. Lentivirus packaging cell line HEK-293T and gastric cancer cell line SGC-7901 were preserved in our laboratory and were cultured in Dulbecco's modified Eagle's medium (DMEM; Invitrogen) supplemented with $10 \%$ fetal calf serum (FCS) (HyClone; Thermo Scientific), $100 \mathrm{U} / \mathrm{ml}$ penicillin and $0.1 \mathrm{mg} / \mathrm{ml}$ streptomycin. Cells were maintained at $37^{\circ} \mathrm{C}$ in an incubation chamber with $5 \% \mathrm{CO}_{2}$.

Lentivirus production and double-labeled cell line construction. Lentivirus plasmid pWPT-GFP, pMD2.G, psPAX2 and pLentiCMV-Puro-LUC were purchased from Addgene (catalog nos. 12255, 12259, 12260 and 17477); lentivirus packaging plasmids Delta 8.9 and VSVG were kindly provided by Professor C. Lois. The day before transduction, $4 \times 10^{6}$ low-passage and healthy $293 \mathrm{~T}$ cells were seeded in a $10 \mathrm{~cm}$ dish. When cells obtained $60-70 \%$ confluency, the transduction was done by introducing pLenti-CMV-Puro-LUC, delta 8.9 and VSVG into $293 \mathrm{~T}$ cells using Lipofectamine 2000 (Invitrogen) according to manufacturer's instructions. At $48 \mathrm{~h}$ post-transduction, the supernatant was collected, filtered and stored at $-80^{\circ} \mathrm{C}$. The day prior to infection, $5 \times 10^{5}$ SGC-7901 cells were plated in the 6-well plate, while lentivirus stocks and polybrene ( $8 \mu \mathrm{g} / \mathrm{ml}$; Millipore) were fully mixed in complete growth medium. The plate was then centrifuged at $1200 \mathrm{xg}$ for $90 \mathrm{~min}$ at $32^{\circ} \mathrm{C}$. The medium was changed the next day; puromycin $(1.0 \mu \mathrm{g} / \mathrm{ml}$; Sigma) was added at $48 \mathrm{~h}$ post-transduction and maintained for $\sim 2$ weeks. As for GFP lentivirus packing and transduction, procedures were almost the same as described previously, except for the plasmids we used (pWPT-GFP, pMD2.G, psPAX2). Transduction efficiency was monitored by a converted fluorescence microscope (IX51; Olympus), and fluorescenceactivated cell sorting (FACS; BD Biosciences) was used to collect the GFP-positive cells.

In vitro imaging of SGC7901-Luc-GFP. Different number of cells for SGC7901-Luc-GFP was used in bioluminescence and fluorescence imaging. Before imaging, SGC7901-Luc-GFP cells were placed in a 96-well plate, and a 2-fold serial dilution was made. Then, the culture plate was placed in a light-tight chamber equipped with a halogen light source (IVIS Kinetics; Caliper Life Science). For GFP imaging, the whole image was acquired for a $5 \mathrm{sec}$ exposure with a GFP filter set and excitation wavelength at $465 \mathrm{~nm}$. For bioluminescence imaging, $5 \mu \mathrm{l}$ D-luciferin (150 $\mu \mathrm{g} / \mathrm{ml}$; Caliper Life Science) was added $1 \mathrm{~min}$ prior to imaging, and a bioluminescent signal was then measured for a $180 \mathrm{sec} / 3 \mathrm{~min}$ acquisition. Regions of interest (ROIs) were drawn and light intensity was quantified using Living Image version 4.2 Software. Fluorescent or bioluminescent intensity was presented as (photons $\left./ \mathrm{sec} / \mathrm{cm}^{2} / \mathrm{sr}\right) \div\left(\mu \mathrm{W} / \mathrm{cm}^{2}\right)$ or photons/ $\mathrm{sec} / \mathrm{cm}^{2} / \mathrm{sr}$, respectively.

Orthotopic gastric cancer animal model. Female athymic BALB/c nude mice, 6 to 7 weeks old, were obtained from the Animal Experimental Center of Slaccas (Shanghai, China). The animals were maintained in a laminar airflow cabinet under specific pathogen-free conditions and allowed free access to standard laboratory food and water as well as $12 \mathrm{~h}$ of light and a dark cycle per day. All animal protocols were approved by the Animal Welfare and Ethics Committee of the Fourth Military Medical University (FMMU).

Animals were anesthetized with ketamine and xylazine, and a 2-3 cm upper left abdominal incision was cautiously made. The stomach was carefully exposed, and 5x106 SGC7901Luc-GFP cells suspended in $50 \mu 1$ DMEM were orthotopically inoculated into the sub-serosa layer of the stomach wall using 30-G needles (Becton; Dickinson and Company). The microinjection site was pressed with a cotton swab for several minutes in case of cell suspension effusion resulting in peritoneal cavity implantation. The stomach was then prudently returned to the peritoneal cavity, and the abdominal wall and skin were closed with Prolene suture. All of the procedures were done with the aid of a stereomicroscope (SZX7; Olympus).

In vivo bioluminescence imaging and drug response monitoring. Mice were anesthetized with isofluorane and subsequently received filter-sterilized D-luciferin solution $(150 \mathrm{mg} / \mathrm{kg}$ body weight) intraperitoneally. The mice were then placed in the chamber with right lateral recumbency. Ten minutes after D-luciferin injection, BLI was implemented for obtaining the peak intensity signal. The bioluminescent intensities of the ROIs were measured and represented as previously described.

Seven days after tumor incubation, the mice were assigned to receive docetaxel ( $8 \mathrm{mg} / \mathrm{kg}$; Taxotere, Sanofi-Aventis) or $0.9 \%$ saline treatment once a week. Mouse composition in a different group was kept balanced according to the bioluminescent intensity prior to drug admiration. Drug response was monitored by BLI.

\section{Three-dimensional reconstruction via bioluminescence tomo-} graphy. Random mouse models from the treated group were imaged to estimate the drug response sensitivity after treatment at an appropriate time point through bioluminescence tomography. In a similar way of bioluminescence imaging, the D-luciferin solution (150 mg/kg body weight) was injected into the mouse peritoneal cavity (i.p.) 10 min prior to bioluminescence tomography, and then the mouse was placed onto the warmed stage inside the light-tight chamber and received continuous exposure under gaseous anesthesia (isoflurane $2 \%$, oxygen 0.3 liters/min) to sustain sedation during imaging. Micro-CT slices of the mouse models were accurately segmented through intraperitoneal Iohexol accumulative contrast enhancement to obtain the anatomical information (25) such as for the heart, lungs, liver, stomach, and kidneys using commercially available software Amira 4.1.1 (Mercury Computer System, Inc.). In this heterogeneous tumor-bearing mouse model, all optical parameters with a $620 \mathrm{~nm}$ wavelength (shown in Table I) were adopted for different organs and tissues. Bioluminescence tomography was quantified in units of total power (nW) inside 
Table I. Optical parameters of the mouse organs (26).

\begin{tabular}{llllllll}
\hline Material & Adipose & Heart & Lungs & Liver & Stomach & Kidneys & Spleen \\
\hline $620 \mathrm{~nm}$ & & & & & & & \\
$\mu_{\alpha}\left[\mathrm{mm}^{-1}\right]$ & 0.0086 & 0.1382 & 0.4596 & 0.8291 & 0.0261 & 0.1550 & 0.8293 \\
$\mu_{\mathrm{s}}^{\prime}\left[\mathrm{mm}^{-1}\right]$ & 1.2584 & 1.0769 & 2.2651 & 0.7356 & 1.5492 & 2.5329 & 0.7356 \\
\hline
\end{tabular}

A

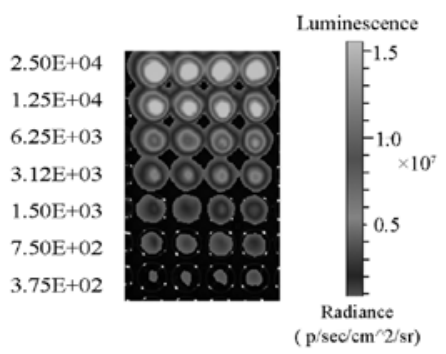

C

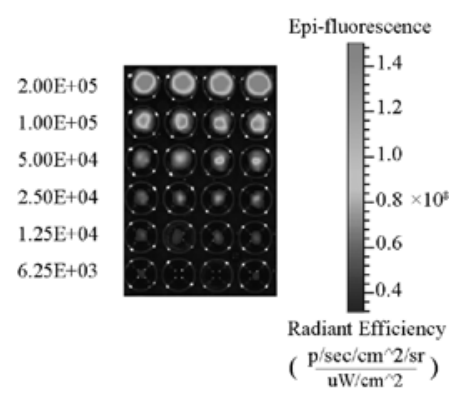

B

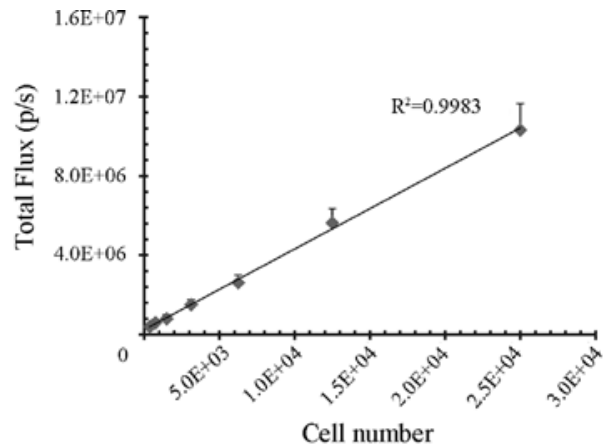

$\mathrm{D}$

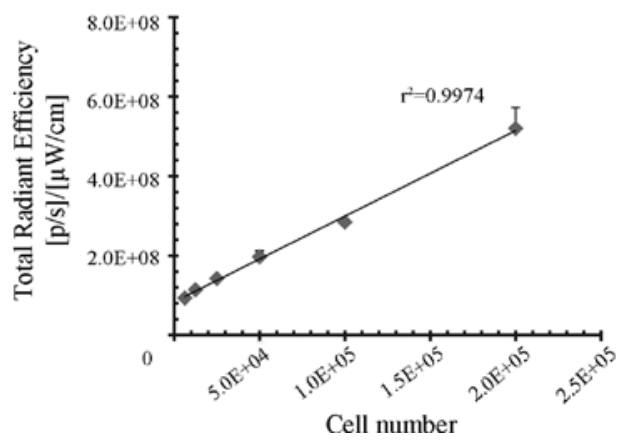

Figure 1. Optical characteristics of SGC7901-Luc-GFP. (A and C) Cells were 2-fold serially diluted, then seeded in a black 96-well plate. In vitro bioluminescence and fluorescence imaging were conducted. (B and D) Linear regression analysis showed a good correlation between cell number and mean BLI or fluorescent intensity $\left(\mathrm{R}^{2}=0.9983, \mathrm{r}^{2}=0.9974\right)$.

the living mouse, where the reconstruction algorithm based on the finite element method (FEM) was adopted in our studies of gastric cancer reconstruction in vivo $(26,27)$.

Histological studies. Mice were sacrificed at the end of the experiment. The primary stomach lesion was excised. The tissues were fixed in formalin, embedded in paraffin and contiguous sections $(4 \mu \mathrm{m})$ were obtained for hematoxylin and eosin $\mathrm{H} \& \mathrm{E}$ analysis. Frozen $(8 \mu \mathrm{m})$ sections were also prepared at $-20^{\circ} \mathrm{C}$ to confirm GFP existence (CM1900; Leica), and fluorescent images were collected via fluorescent microscope (BX51; Olympus). The slices were analyzed by two independent pathologists at our laboratory.

Statistical analysis. Data are expressed as the mean \pm standard deviation (SD). A Student's t-test was used to assess the P-value. Analysis of variance (ANOVA) for repeated measures was implemented to analyze drug efficacy. Regression plots were used to describe the correlation. PASW Statistics 18.0 (SPSS Inc., IBM Corp.) was used for statistical analysis. Differences between groups were considered as statistically significant at $\mathrm{P}<0.05$.

\section{Results}

Characteristics of the transformed gastric cell line SGC7901Luc-GFP. The stable expression of Luciferase and GFP in the gastric cancer cell line is a prerequisite for intensive research with an advanced molecular imaging approach. After being sequentially infected by luciferase and GFP contained lentivirus, the gastric cell line SGC7901-Luc-GFP was developed. To determine whether or not the cell characteristics were influenced by the virus infection process, we compared cell proliferation and morphological differences between the transformed clones and their correspondent parental strains. No obvious changes were observed in the in vitro growth pattern; SGC7901-Luc-GFP shared a similar morphological appearance as its parental cells with $90 \%$ GFP positive cells (data not shown). To compare the photons generating the ability between transformed and parental cells, we treated both kinds of cells with substrate D-luciferin. The subsequent results showed a steady growth in bioluminescent activity accompanied by an increasing cell number for SGC7901-Luc-GFP; however, no bioluminescence was detected in parental SGC7901 despite a cell number increase (data not shown). Furthermore, we serially diluted the cells and 

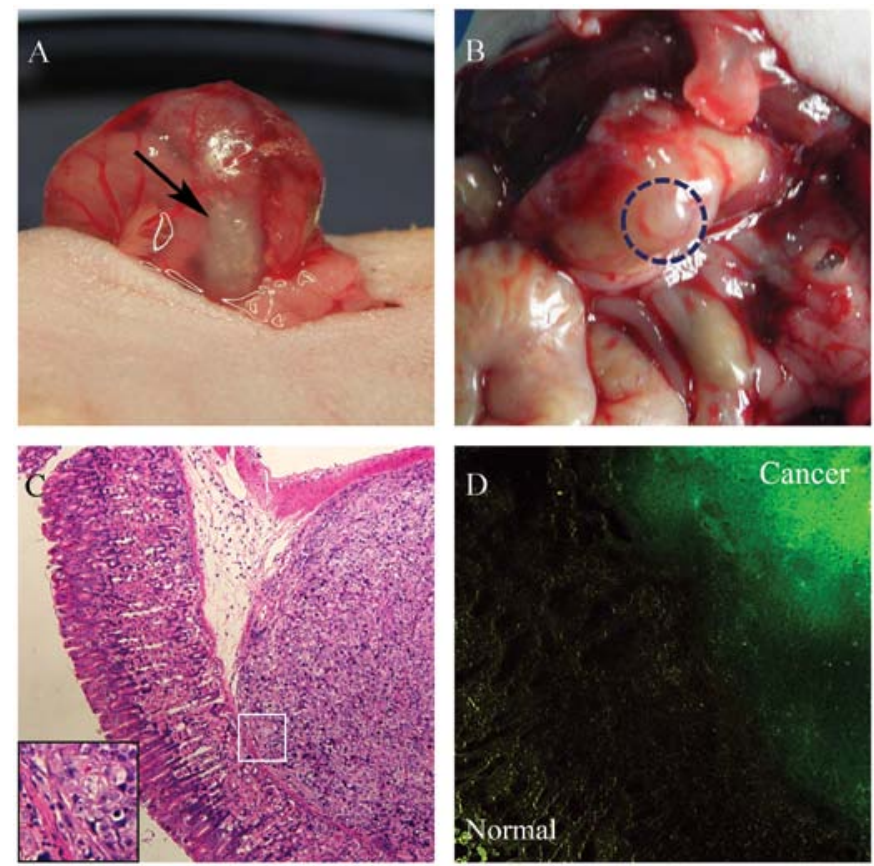

Figure 2. Macroscopic views of the orthotopic cancer model. (A) SGC7901Luc-GFP cells $\left(5 \times 10^{6}\right)$ were incubated in the sub-serosa layer of the stomach; injection site (black arrow). (B) Mice were sacrificed 12 days after orthotopic implantation. The stomach was exposed and the primary tumor lesion was clearly presented (dashed cycle). (C) Tissues were fixed, embedded and analyzed with an H\&E stain (magnification, x100, thumbnail x200). (D) GFP was confirmed by fluorescent microscope after standard frozen section treatment (magnification, x200).

assessed the accuracy of BLI as well as fluorescent imaging as indicators of cell number. Correlation analysis revealed a linear relationship between bioluminescent intensity and cell number $\left(\mathrm{R}^{2}=0.9983\right)$ with a minimum detection of 375 cells per well (Fig. 1A and B). As for GFP, a similar trend was also observed $\left(r^{2}=0.9974\right)$, but due to the auto-fluorescence, GFP had high signal/noise ratio making it ineffectual in quantifying a small number of cells (Fig. 1C and D).

Mouse orthotopic model of human gastric cancer. Under the standard surgical procedures, 5x10 6 SGC7901-Luc-GFP cells were injected into the sub-serosa layer of the stomach wall (Fig. 2A). There were no complications recorded during the entire operation process and all mice recovered uneventfully from anesthesia. In order to confirm successful implantation, two mice were sacrificed 12 days later. Postmortem examination showed that both mice had developed a stomach tumor on the gastric surface (Fig. 2B). H\&E staining showed that SGC7901-Luc-GFP cells were extensively implanted in the stomach wall with a poorly differentiated and highly mitotic phenotype. Frozen section fluorescent images showed the GFP tumor position in sharp contrast to the normal epithelium (Fig. 2C and D).

Tumor progression and regression monitored by in vivo bioluminescence imaging. In order to explore whether or not our modified cancer cell line could track tumor progression and regression after treatment in vivo, we recorded the dynamic changes in light intensity for each mouse using the bioluminescence imaging system. A bioluminescent signal was detected
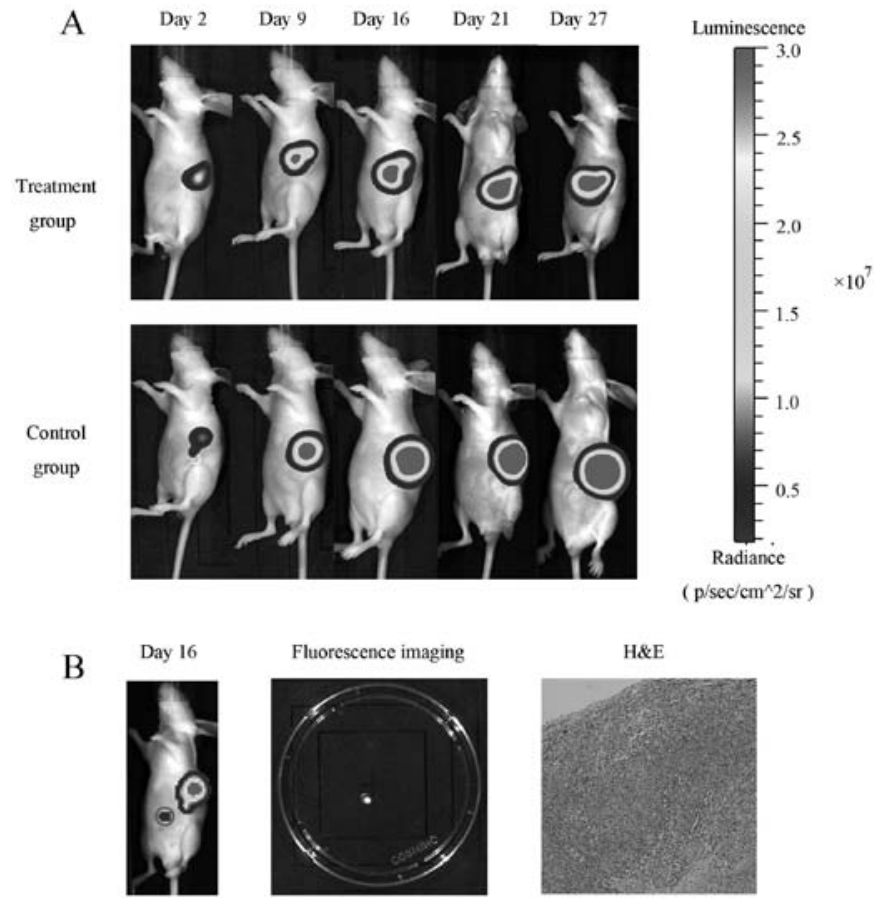

Figure 3. Tumor progression and drug response monitored by in vivo bioluminescence imaging. Bioluminescence variation was recorded on a weekly basis. (A) Serial images at different time-points for the representative mouse in the control and treatment group. (B) Probable local metastasis (cycle) was recorded on day 16 , and the metastasis was resected and confirmed by in vitro GFP imaging and H\&E staining.

2 days after incubation, and signal distribution was mainly focused on the stomach region. On day 7 , mice were assigned to receive docetaxel treatment $(8 \mathrm{mg} / \mathrm{kg})$ or vehicle $(0.9 \%$ saline $)$ via tail vein injection weekly. The signal existed through the entire observation period both in the control and treatment group (Fig. 3A). On day 16, additional signals were recorded beside the primary signal in 2 mice, indicating probable local metastasis; subsequent GFP imaging and H\&E analysis confirmed our assumptions (Fig. 3B). Continuous data revealed an exponential increase in bioluminescent activity that was correlated with tumor progression $\left(\mathrm{R}^{2}=0.986\right.$, Fig. 4A). Further repeated measurement analysis showed statistical significance between the treatment and control group. However, we noted that docetaxel alone could not eradicate the tumor $(\mathrm{P}<0.05$, Fig. 4B). At the end of the experiment (day 27), all of the mice were cachexia and inactive. The tumors were resected and weighed after the mice were sacrificed. We then found a good linear correlation between tumor weight and bioluminescent activity $\left(\mathrm{R}^{2}=0.9795\right.$, Fig. $\left.4 \mathrm{C}\right)$. Moreover, docetaxel treatment delayed tumor growth compared with the saline control group (Fig. 4D).

Three-dimensional reconstruction of gastric cancer. Through our previously described accumulative contrast enhancement method, good contrast for different organs in the mice was achieved. The outlines of various organs including the heart, lungs, liver, stomach and tumors were carefully segmented in every micro-CT slice. The low background for the bioluminescent signals provided substantially greater sensitivity. Meanwhile, our original optical data showed a strong 
A

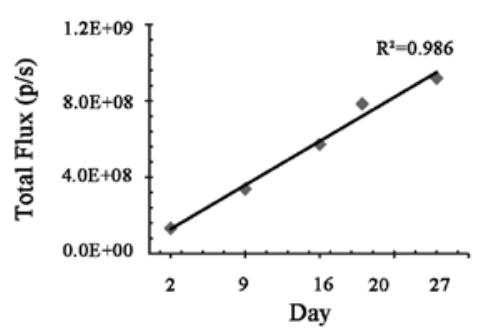

$\mathrm{C}$

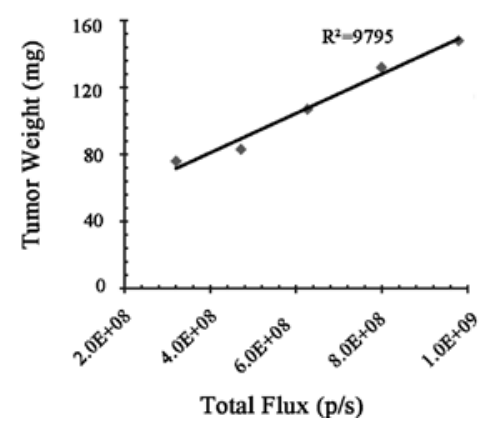

B

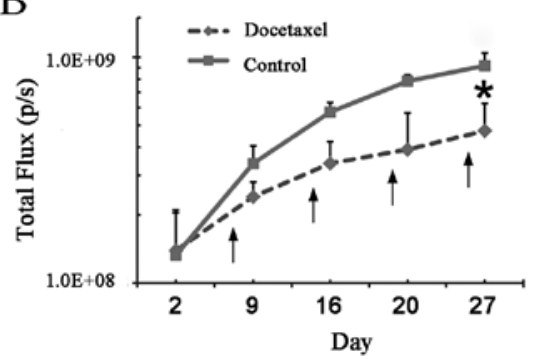

D

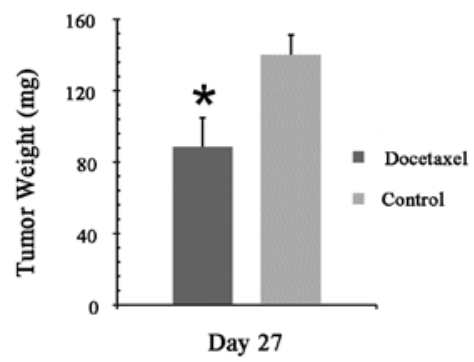

Figure 4. Statistical analysis of bioluminescence and tumor weight. (A) Linear relationship between bioluminescence and tumor progression ( $\mathrm{R}^{2}=0.986$ ). (B) Mean bioluminescence for the treatment and control group are plotted at indicated time intervals (arrow); the treatment group showed a slower signal increase than for the controls $(\mathrm{P}<0.05)$. (C) The weight of the tumor $(\mathrm{mg})$ was correlated with bioluminescent activity for each mouse $\mathrm{R}^{2}=0.9795$. $(\mathrm{D})$ Significant effect of docetaxel on tumor weight in comparison with the saline controls $\left(\mathrm{N}=5\right.$ per group; $\left.{ }^{*} \mathrm{P}<0.05\right)$.

A
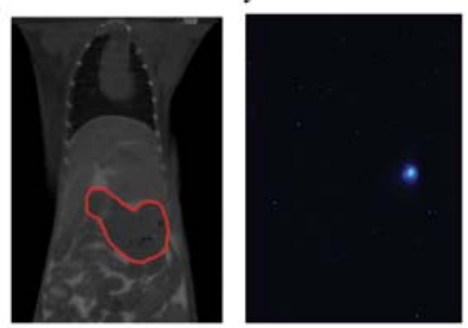

Day 21

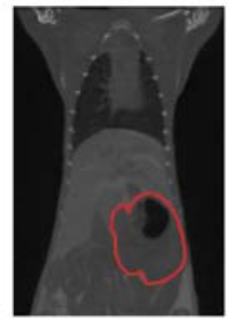

B

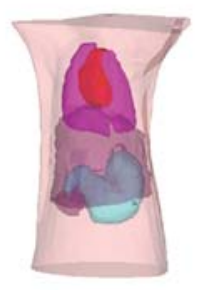

Day 2

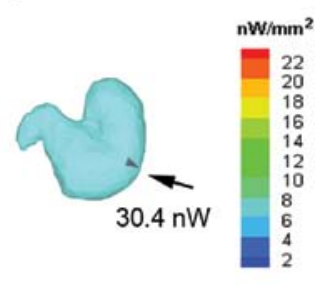

Day 21
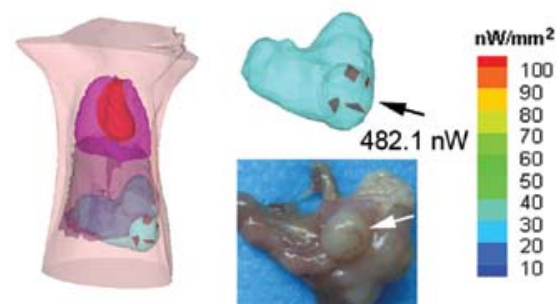

Figure 5. Reconstruction total power (nW) of the gastric tumor on day 2 and 21, respectively. (A) Typical micro-CT slices after contrast enhancement and representative original optical data (red cycle, stomach region). (B) Reconstruction results in heterogeneous mouse models and the amplified images of the reconstructed source and actual stomachs. A tumor lesion is shown in the thumbnail figure, where the tumor distribution is almost the same as the reconstruction results (black arrow, reconstructed light sources; white arrow, actual tumor appearance; red tetrahedral area, reconstructed source).

signal and high signal-to-noise ratio (Fig. 5A). The restructured image for micro-CT distinctly displayed a stereoscopic shape of the stomach with the tumor. The tomographic reconstruction of the light resource was also ideally co-registered. On day 2, the signal located in the greater curvature of the stomach was consistent with the injection site; on day 21 , the light distribution of the same source was mainly focused on the tumor lesion. The total power of BLT reconstruction was 30.4 and $482.1 \mathrm{nW}$ on day 2 and 21, respectively. Moreover, our morphological reconstruction of the affected stomach was similar to the autopsy observation (Fig. 5B).

\section{Discussion}

In this study, we developed a stable expression Firefly luciferase and GFP gastric cancer cell line, SGC7901-Luc-GFP. 
BLI exhibited superior sensitivity and unsurpassed signal/ noise ratios in in vitro and in vivo imaging, which is consistent with previous studies $(28,29)$. We also found a robust correlation between tumor weight and bioluminescence activities. In addition, our transformed cell expressed GFP at a high level, which facilitates postmortem observation since no oxygen or ATP is needed for imaging. Thus, it provides a more accurate assessment of tumor spread. In our research, probable metastatic tissue was resected and confirmed by ex vivo GFP imaging.

The preclinical animal cancer model is the most informative and effective platform to obtain relevant clinical data and evaluate anticancer therapeutics. Previous studies have clearly showed that agent evaluation or a screen carried on an ectopic model must be followed up by relevant orthotopic models (17). Under the clinical identical microenvironment, tumors were modulated by cellular molecules released by host cells (30). In the present study, transformed cells were planted within the orthotopic environment to guarantee, to the maximum extent, that tumor evaluation and potential metastasis in research were the most precise representations of what actually happens in human cancer. It is acknowledged that only a small amount of cells could flee from the primary site and eventually metastasize. The properties of metastatic cells vary considerably from those at the primary site (31-33). We incidentally recorded spontaneous metastasis on day 16. This orthotopic model was obviously superior to experimental metastasis models.

In the field of in vivo gastric cancer research, BLI technology has several noticeable advantages over traditional methods as summarized below. Firstly, as a non-invasive imaging technique, BLI successfully enables data collection from serial images from the identical animal, which avoids the unnecessary usage of large quantities of animals at different time intervals in cancer studies. Secondly, BLI offers a relative assessment of treatment efficacy compared with other detection means, since it only represents the metabolically active tumor cells rather than volumetric measurement of the tumor mass (9). Thirdly, BLI can serve as an indicator of successful implantation of tumor cells. This indicative feature of BLI rules out some faulty responses of the drug which are possibly due to unsuccessful implantation of the xenograft. It greatly benefits efficacy evaluation of the drug for those cancers that cannot be directly detected or measured, such as gastric cancer. In this current research, successful incubation of tumor cells was immediately observed with in vivo imaging of the mice. The outcome of docetaxel treatment was tracked by BLI. Furthermore, the BLI technique helped to eliminate statistical variations, thus the mouse composition in a different group was kept balanced according to the bioluminescence intensity before drug administration.

Commercially available BLI only collects the tumor signal in a surface-weighted manner on a two-dimensional plane $(13,34)$, which means a weaker source near the surface would have identical light intensity with a stronger source in deep tissue (35). Therefore, we tried to monitor the progression of gastric tumors by home-developed BLT system for the first time. BLT could partially overcome this limitation and improve data accuracy (36). Moreover, it conveys information of the anatomical location as well as the bioluminescence signal of the tumor in a stereoscopic view. In our previous studies, BLT was able to reconstruct three-dimensional bioluminescence distribution and provide accurate quantification of the source signal on the basis of appropriate reconstruction algorithm combination of the micro-CT guide (27,36-41). In this study, we utilized BLT to reconstruct morphological appearance and quantify the signal intensity on day 2 and day 21 , respectively. BLT quantitative results were more favorable than BLI results since they were competent in magnifying and improving the results shown by BLI, which was more rational for 3D space distribution integration of tumor signals. In addition, the reconstruction appearance of the affected stomach was quite similar to the post-mortem examination results. Collectively, BLT technique provides new alternative solution for future gastric cancer studies.

Nevertheless, this study has three major limitations. Firstly, our cancer model was developed on immunoincompetent mice, which determines that it should be cautiously generalized when applied to immunoregulation agents. Secondly, the post-processing of semi-automatic segmentation used in the study is relatively labor-intensive and time-consuming, which indicates that our BLT data are not sufficient. Therefore, a more manageable and convenient post-processing approach for data collection is expected in further relevant studies. Another limitation addresses BLT reconstruction signal disturbance as tetrahedral shape, which was possibly caused by the finite dissection of the tetrahedral elements. Some tetrahedrons which represent the bioluminescent intensity are discontinuous and isolated in most reconstructed mice. As a result, some intensive studies on this issue are strongly recommended.

The current study developed novel bioluminescencebased preclinical gastric cancer models which enable superior, noninvasive gastric cancer monitoring. As the first group to employ a novel BLT in gastric cancer, we demonstrated that BLT provided more comprehensive information of the cancerous lesion. The implementation of the opticalimaging-based approach may open new avenues for drug evaluations and preclinical oncological investigations in gastric cancer.

\section{Acknowledgements}

The study was supported by combined grants from the National Natural Science Foundation of China (Nos. 81090270, 81090273, and 30873005), the National Key and Basic Research Development Program of China (No. 2010CB529302), and the National Municipal Science and Technology Project (2009ZX09103-667 and 2009ZX09301-009-RC06). Our special thanks go to Professor Carlos Lois for his generously offered plasmids, without which the current study could not have been completed.

\section{References}

1. Jemal A, Bray F, Center MM, Ferlay J, Ward E and Forman D: Global cancer statistics. CA Cancer J Clin 61: 69-90, 2011.

2. De Vita F, Giuliani F, Galizia G, et al: Neo-adjuvant and adjuvant chemotherapy of gastric cancer. Ann Oncol 18 (Suppl 6): vi120-vi123, 2007.

3. Welsh DK and Kay SA: Bioluminescence imaging in living organisms. Curr Opin Biotechnol 16: 73-78, 2005. 
4. Weissleder R and Pittet MJ: Imaging in the era of molecular oncology. Nature 452: 580-589, 2008.

5. Buchhorn HM, Seidl C, Beck R, et al: Non-invasive visualisation of the development of peritoneal carcinomatosis and tumour regression after $\mathrm{Bi}-213$-radioimmunotherapy using bioluminescence imaging. Eur J Nucl Med Mol Imaging 34: 841-849, 2007.

6. Zhao H, Doyle TC, Coquoz O, Kalish F, Rice BW and Contag CH: Emission spectra of bioluminescent reporters and interaction with mammalian tissue determine the sensitivity of detection in vivo. J Biomed Opt 10: 41210, 2005.

7. Angst E, Chen M, Mojadidi M, Hines OJ, Reber HA and Eibl G: Bioluminescence imaging of angiogenesis in a murine orthotopic pancreatic cancer model. Mol Imaging Biol 12: 570-575, 2010.

8. Snoeks TJA, Lowik C and Kaijzel EL: 'In vivo' optical approaches to angiogenesis imaging. Angiogenesis 13: 135-147, 2010.

9. Jenkins DE, Oei Y, Hornig YS, et al: Bioluminescent imaging (BLI) to improve and refine traditional murine models of tumor growth and metastasis. Clin Exp Metastasis 20: 733-744, 2003.

10. Chen L, Yang S, Jakoncic J, Zhang JJ and Huang X-Y: Migrastatin analogues target fascin to block tumour metastasis. Nature 464 1062-1135, 2010

11. Pichler A,Zelcer N, Prior JL, Kuil AJ and Piwnica-Worm D: In vivo RNA interference-mediated ablation of MDR1 P-glycoprotein. Clin Cancer Res 11: 4487-4494, 2005.

12. Hajitou A, Trepel M, Lilley CE, et al: A hybrid vector for liganddirected tumor targeting and molecular imaging. Cell 125: 385-398, 2006.

13. Zeamari S, Rumping G, Floot B, Lyons S and Stewart FA: In vivo bioluminescence imaging of locally disseminated colon carcinoma in rats. Br J Cancer 90: 1259-1264, 2004

14. Edinger M, Cao YA, Verneris MR, Bachmann $\mathrm{MH}$, Contag $\mathrm{CH}$ and Negrin RS: Revealing lymphoma growth and the efficacy of immune cell therapies using in vivo bioluminescence imaging. Blood 101: 640-648, 2003.

15. Paroo Z, Bollinger RA, Braasch DA, et al: Validating bioluminescence imaging as a high-throughput, quantitative modality for assessing tumor burden. Mol Imaging 3: 117-124, 2004

16. Wilmanns C, Fan D, Obrian CA, Bucana CD and Fidler IJ: Orthotopic and ectopic organ environments differentially influence the sensitivity of murine colon carcinoma cells to doxorubicin and 5-fluorouracil. Int J Cancer 52: 98-104, 1992.

17. Killion JJ, Radinsky R and Fidler IJ: Orthotopic models are necessary to predict therapy of transplantable tumors in mice. Cancer Metastasis Rev 17: 279-284, 1998

18. Hoffman RM: Orthotopic metastatic mouse models for anticancer drug discovery and evaluation: a bridge to the clinic. Invest New Drugs 17: 343-359, 1999.

19. Bibby MC: Orthotopic models of cancer for preclinical drug evaluation: advantages and disadvantages. Eur J Cancer 40 $852-857,2004$

20. McCarty MF, Takeda A, Stoeltzing O, et al: ZD6126 inhibits orthotopic growth and peritoneal carcinomatosis in a mouse model of human gastric cancer. Br J Cancer 90: 705-711, 2004.

21. Nakajima TE, Yanagihara K, Takigahira M, et al: Antitumor effect of SN-38-releasing polymeric micelles, NK012, on spontaneous peritoneal metastases from orthotopic gastric cancer in mice compared with irinotecan. Cancer Res 68: 9318-9322, 2008.

22. Sumida T, Kitadai Y, Shinagawa K, et al: Anti-stromal therapy with imatinib inhibits growth and metastasis of gastric carcinoma in an orthotopic nude mouse model. Int J Cancer 128: 2050-2062, 2011.
23. Wang G, Hoffman E, McLennan G, Wang L, Suter M and Meinel J: Development of the first bioluminescent CT scanner. Radiology 229: 566, 2003.

24. Wang G, Li Y and Jiang M: Uniqueness theorems in bioluminescence tomography. Med Phys 31: 2289-2299, 2004.

25. Liu JTJ, Li X, Yang X, Chen X, Chen Y and Zhou Y: Detecting metastasis of gastric carcinoma using high-resolution micro-CT system: in vivo small animal study. Proc SPIE Medical Imaging 7965: 796501-779656, 2011

26. Alexandrakis G, Rannou F and Chatziioannou A: Tomographic bioluminescence imaging by use of a combined optical-PET (OPET) system: a computer simulation feasibility study. Phys Med Biol 50: 4225-4242, 2005.

27. Liu J, Wang Y, Qu X, et al: In vivo quantitative bioluminescence tomography using heterogeneous and homogeneous mouse models. Opt Express 18: 13102-13113, 2010.

28. Edinger M, Cao YA, Hornig YS, et al: Advancing animal models of neoplasia through in vivo bioluminescence imaging. European Eur J Cancer 38: 2128-2136, 2002.

29. Vilalta M, Degano IR, Bago J, et al: Biodistribution, long-term survival, and safety of human adipose tissue-derived mesenchymal stem cells transplanted in nude mice by high sensitivity noninvasive bioluminescence imaging. Stem Cells Dev 17: 993-1003, 2008.

30. Bogenrieder T and Herlyn M: Axis of evil: molecular mechanisms of cancer metastasis. Oncogene 22: 6524-6536, 2003

31. Francia G, Cruz-Munoz W, Man S, Xu P and Kerbel RS: Mouse models of advanced spontaneous metastasis for experimental therapeutics. Nat Rev Cancer 11: 135-141, 2011.

32. Fidler IJ and Balch CM: The biology of cancer metastasis and implications for therapy. Curr Probl Surg 24: 129-209, 1987.

33. Ito $H$, Hatori $M$, Kinugasa $Y$, Irie $T$, Tachikawa $T$ and Nagumo $M$ : Comparison of the expression profile of metastasis-associated genes between primary and circulating cancer cells in oral squamous cell carcinoma. Anticancer Res 23: 1425-1431, 2003.

34. Doubrovin M, Serganova I, Mayer-Kuckuk P, Ponomarev V and Blasberg RG: Multimodality in vivo molecular-genetic imaging. Bioconjug Chem 15: 1376-1388, 2004.

35. El-Deiry WS, Sigman CC and Kelloff GJ: Imaging and oncologic drug development. J Clin Oncol 24: 3261-3273, 2006.

36. Luker GD and Luker KE: Optical imaging: Current applications and future directions. J Nucl Med 49: 1-4, 2008.

37. Liu J, Chen D, Li X, et al: In vivo quantitative reconstruction studies of bioluminescence tomography: effects of peak-wavelength shift and model deviation. IEEE Trans Biomed Eng 57: 2579-2582, 2010.

38. Lu Y, Machado HB, Bao Q, Stout D, Herschman H and Chatziioannou AF: In vivo mouse bioluminescence tomography with radionuclide-based imaging validation. Mol Imaging Biol 13: 53-58, 2011

39. Lv Y, Tian J, Cong W, et al: A multilevel adaptive finite element algorithm for bioluminescence tomography. Opt Express 14: 8211-8223, 2006.

40. Cong WX, Wang G, Kumar D, et al: Practical reconstruction method for bioluminescence tomography. Opt Express 13 6756-6771, 2005 .

41. Wang G, Cong W, Durairaj K, et al: In vivo mouse studies with bioluminescence tomography. Opt Express 14: 7801-7809, 2006. 